\title{
Tetrandrine induces apoptosis and triggers a caspase cascade in U2-OS and MG-63 cells through the intrinsic and extrinsic pathways
}

\author{
LI-JIANG TAO, XIN-DIE ZHOU, CHENG-CHUN SHEN, \\ CHENG-ZHEN LIANG, BING LIU, YIQING TAO and HUI-MIN TAO \\ Department of Orthopedics, Second Affiliated Hospital, School of Medicine, \\ Zhejiang University, Hangzhou, Zhejiang 310009, P.R. China
}

Received May 16, 2013; Accepted October 16, 2013

DOI: $10.3892 / \mathrm{mmr} .2013 .1761$

\begin{abstract}
Although neoadjuvant chemotherapy has improved the survival rate of osteosarcoma patients, drug resistance remains a predominant obstacle to improving efficacy and necessitates the development of novel chemotherapeutical agents. The aim of this study was to investigate whether tetrandrine (TET) induces apoptosis in the U-2OS and MG-63 osteosarcoma cell lines and to further determine the underlying mechanism. This study investigated the effects of TET on osteosarcoma in vitro. To examine the antitumor effects of TET on osteosarcoma, the two osteosarcoma cell lines were treated with TET and subjected to apoptosis assays. The results revealed that TET induced the apoptosis of osteosarcoma cells in a time- and dose-dependent manner. Furthermore, the apoptosis of osteosarcoma cells was accompanied by increased cytochrome $c$ (Cyto-C), apoptotic protease-activating factor (Apaf)-1, Bid and Bax activation and reduced Bcl-2 and Bcl-xl activation, demonstrating that the apoptosis may have occurred through the mitochondrial pathway. In conclusion, the results suggest that TET is a promising agent for osteosarcoma therapy.
\end{abstract}

\section{Introduction}

Osteosarcoma, the most common type of malignant bone tumor, occurs predominantly in children and adolescents (1-3). Although the combination of surgery and aggressive adjuvant chemotherapy has largely improved the 5-year survival rates of osteosarcoma patients, the acquisition of resistance to the cytotoxic effects of chemotherapy and adverse effects have emerged as significant obstacles to effective cancer treatment (4-6). In

Correspondence to: Professor Hui-Min Tao, Department of Orthopedics, Second Affiliated Hospital, School of Medicine, Zhejiang University, 88 Jie Fang Road, Hangzhou, Zhejiang 310009, P.R. China

E-mail: huimintao_zrgk@163.com

Key words: osteosarcoma, tetrandrine, apoptosis, caspase, intrinsic pathway, extrinsic pathway order to improve treatment efficacy, novel antitumor agents and therapeutic approaches are urgently required.

In recent years, traditional Chinese medicines have been widely used for the treatment of osteosarcoma as promising agents against chemoresistance and the recurrence of malignant diseases $(7,8)$. Tetrandrine (TET), a bisbenzylisoquinoline alkaloid extracted from the roots of Stephania tetrandra S. Moore, has been reported to induce apoptosis in a variety of cancer cells in vitro (9-13).

In this study, the effects of TET on the proliferation and apoptosis of MG-63 and U2-OS human osteosarcoma cells were examined. It was observed that TET inhibited proliferation and induced apoptosis in osteosarcoma cells. Therefore, it may be inferred that TET is a candidate novel anti-osteosarcoma drug.

\section{Materials and methods}

Reagents and antibodies. TET was purchased from Sigma-Aldrich. (St. Louis, MO, USA). The molecular formula of TET is $\mathrm{C}_{38} \mathrm{H}_{42} \mathrm{~N}_{2} \mathrm{O}_{6}$ and it has a molecular weight of 622.75 (Fig. 1). TET was dissolved in $0.01 \mathrm{M} \mathrm{HCl}$ to produce a TET stock solution with the desired concentration. This TET stock solution was stored at $-20^{\circ} \mathrm{C}$ and was freshly diluted in a cell culture medium prior to its use in each experiment. MTT was also purchased from Sigma-Aldrich. Antibodies specific for cytochrome $c$ (Cyto-C), apoptotic protease-activating factor (Apaf)-1,Bid, Bax, Bcl-2 and Bcl-xl were purchased from Abcam (Cambridge, UK). Horseradish peroxidase (HRP)-conjugated goat anti-mouse and HRP-conjugated goat anti-rabbit secondary antibodies were obtained from Pierce (Rockford, IL, USA).

Cells and cell culture. The U-2OS (HTB-96TM, ATCC) and MG-63 (CRL-1427TM, ATCC) human osteosarcoma cell lines were obtained from the cell bank of the Shanghai Institute of Biochemistry and Cell Biology, Chinese Academy of Sciences (Shanghai, China), where they were analyzed and authenticated. These procedures included cross-species checks, DNA authentication and quarantine. The cell lines used in the present study had been cultured for $<6$ months. U2-OS cells were cultured in RPMI-1640 medium (Gibco-BRL, Carlsbad, CA, USA) supplemented with $10 \%$ fetal calf serum (FCS) and 
$1 \%$ penicillin/streptomycin and MG-63 cells were cultured in Dulbecco's modified Eagle's medium (Invitrogen, Carlsbad, CA, USA) containing the same supplements. All cells were cultured at $37^{\circ} \mathrm{C}$ in a humidified atmosphere $\left(5 \% \mathrm{CO}_{2}, 95 \%\right.$ air $)$.

Cell viability assay. The viability of cells treated with TET was measured by an MTT assay. Cells were plated in 96-well plates $(5,000-8,000$ cells/well) and were incubated overnight to allow the cells time to attach. Subsequently, the medium was removed and replaced with a serum-free medium containing varying concentrations of TET $(0-20 \mu \mathrm{M})$ for various periods of time (0-72 h). Following incubation for the designated period of time, $20 \mathrm{ml}$ MTT solution $(5 \mathrm{mg} / \mathrm{ml}$ in PBS) was added to each well and the cells were incubated in a cell incubator (Thermo Fisher Scientific, Waltham, MA, USA). After $4 \mathrm{~h}$, the medium was replaced with $150 \mathrm{ml}$ dimethylsulfoxide. The absorbance at $570 \mathrm{~nm}$ was measured using a Dynatech MR7000 microplate reader (Dynatech Laboratories Inc., Chantilly, VA, USA). Data are the averages of five replicates, and each experiment was performed in triplicate to reduce errors.

Electron microscopy. Treated and untreated cells were observed by transmission electronic microscopy (TEM). Cells were fixed in $4 \%$ glutaraldehyde, postfixed in $1 \%$ osmium tetroxide and embedded in epoxy resin. Serial sections $(0.5 \mu \mathrm{m})$ were stained with uranyl acetate and lead citrate and subsequently observed under a TECNAI 10 transmission electron microscope (Philips, Hillsboro, OR, USA).

Annexin V-fluorescein isothiocyanate (FITC)/propidium iodide (PI) double staining. To quantify the apoptosis of U2-OS and MG-63 cells treated with TET, annexin V-FITC/PI double staining was performed. The cells were seeded in six-well plates $\left(2 \times 10^{5}\right.$ cells/well $)$ and treated with TET $(0-16 \mu \mathrm{M})$ for $24 \mathrm{~h}$. Subsequently, the cells were stained using an annexin V-FITC/PI double-fluorescence apoptosis detection kit (Biouniquer Technology, Nanjing, China), according to the manufacturer's instructions. Samples were analyzed using a FACSCalibur flow cytometer (Becton Dickinson, North Ryde, Australia) within $30 \mathrm{~min}$ of staining.

Caspase activity assay. In order to investigate whether the apoptosis induced by TET was correlated with the activation of caspase enzymes, the activities of the initiator caspase- 9 and effector caspase-3 were analysed. MG-63 and U2-OS cells were seeded on six-well plates $\left(2 \times 10^{5}\right.$ cells/well). Cells were incubated with TET for various periods of time (0-48 h). Following treatment with the designated doses of TET, cells were lysed by freeze/thawing in a lysis buffer. Lysates were clarified by centrifugation (Thermo Fisher Scientific) at $10,000 \mathrm{x}$ g at $4^{\circ} \mathrm{C}$ for $15 \mathrm{~min}$. Protein $(100 \mathrm{mg}$ ) was incubated with $30 \mathrm{ml}$ caspase assay buffer and $2 \mu 1$ of caspase- 3 colorimetric substrate (DEVD-pNA) at $37^{\circ} \mathrm{C}$ for approximately $90 \mathrm{~min}$. Subsequently, the absorbance at $405 \mathrm{~nm}$ was measured using a 96-well microplate reader (Dynatech Laboratories Inc.). Assays were performed in triplicate with various sample dilutions.

Western blot analysis. Cells $\left(2 \times 10^{6}\right)$ treated with 4,8 or $16 \mu \mathrm{M}$ TET were washed with ice-cold PBS. Total protein was subsequently extracted using a total protein extraction

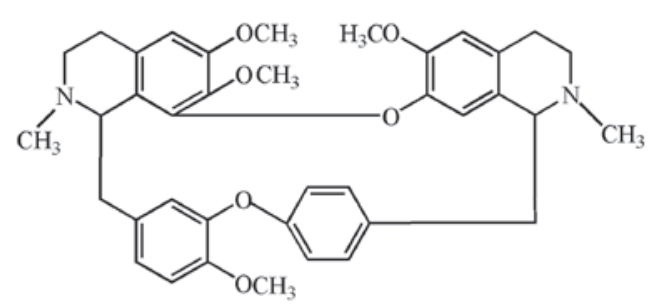

Figure 1. Structural formula of tetrandrine.

A MG-63
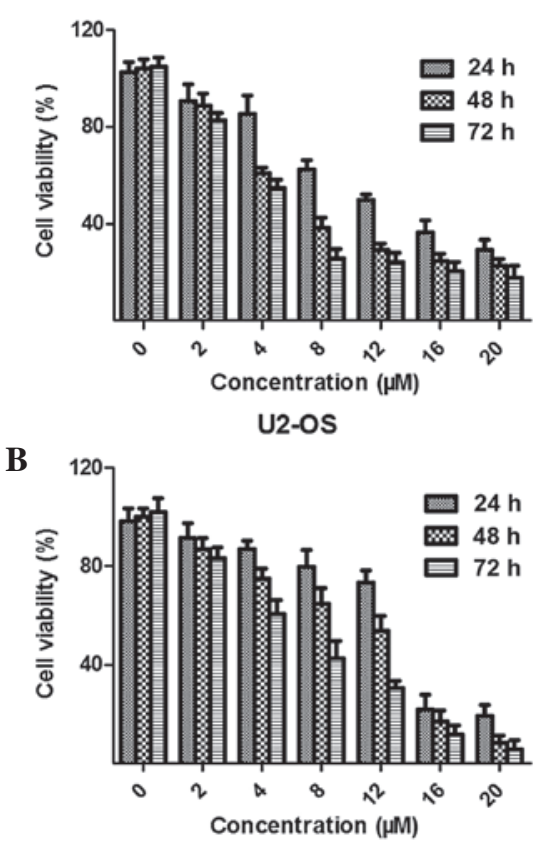

Figure 2. Tetrandrine (TET) inhibits the proliferation of human osteosarcoma cells in a dose- and time-dependent manner. (A) MG-63 and (B) U2-0S cells were exposed to various concentrations of TET for different periods of time and the absorbances at $570 \mathrm{~nm}$ were obtained using a 96-well microtest spectrophotometer following the MTT assay. The inhibition rate was expressed as the percentage of cell inhibition compared with the control. The data are expressed as the mean $\pm \mathrm{SD}$ of triplicate samples.

kit (Millipore, Waltham, MA, USA) and quantified with a bicinchoninic acid protein assay kit (Pierce) according to the manufacturer's instructions. Protein $(\sim 50 \mu \mathrm{g})$ was resolved on denatured $10-15 \%$ SDS-PAGE and subsequently transferred to polyvinylidene fluoride membranes. After blocking for $1 \mathrm{~h}$ in Tris-buffered saline and Tween-20 [TBST (Dakewe, Beijing, China), containing 5\% milk], the membranes were incubated overnight at $4^{\circ} \mathrm{C}$ with antibodies against Bid (anti-Bid antibody; ab32060, Abcam), Bcl-xl (anti-Bcl-xl antibody; ab32370, Abcam), Bax (anti-Bax antibody; ab32503, Abcam), Cyto-C (anti-Cyto-C antibody; ab110325, Abcam), Bcl-2 (anti-Bcl-2 antibody; ab32124, Abcam), Apaf-1 (anti-Apaf-1 antibody; ab32372, Abcam), and $\beta$-actin (anti- $\beta$-actin antibody; Santa Cruz Biotechnology, Santa Cruz, CA, USA). The membranes were incubated with goat anti-mouse IgG-HRP (Pierce) and goat anti-rabbit IgG-HRP (Pierce) secondary antibodies at room temperature for $1 \mathrm{~h}$. Signals were detected using an Enhanced Chemiluminescence kit (GE Healthcare, Shanghai, China) with exposure to X-ray film (Kodak, Hangzhou, China). 
A
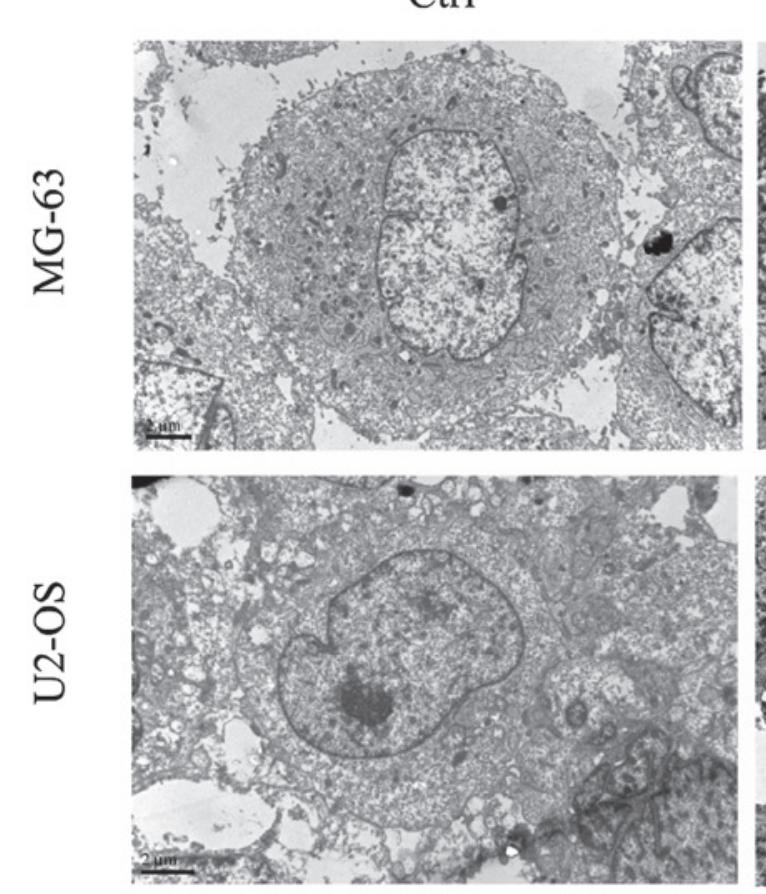

B
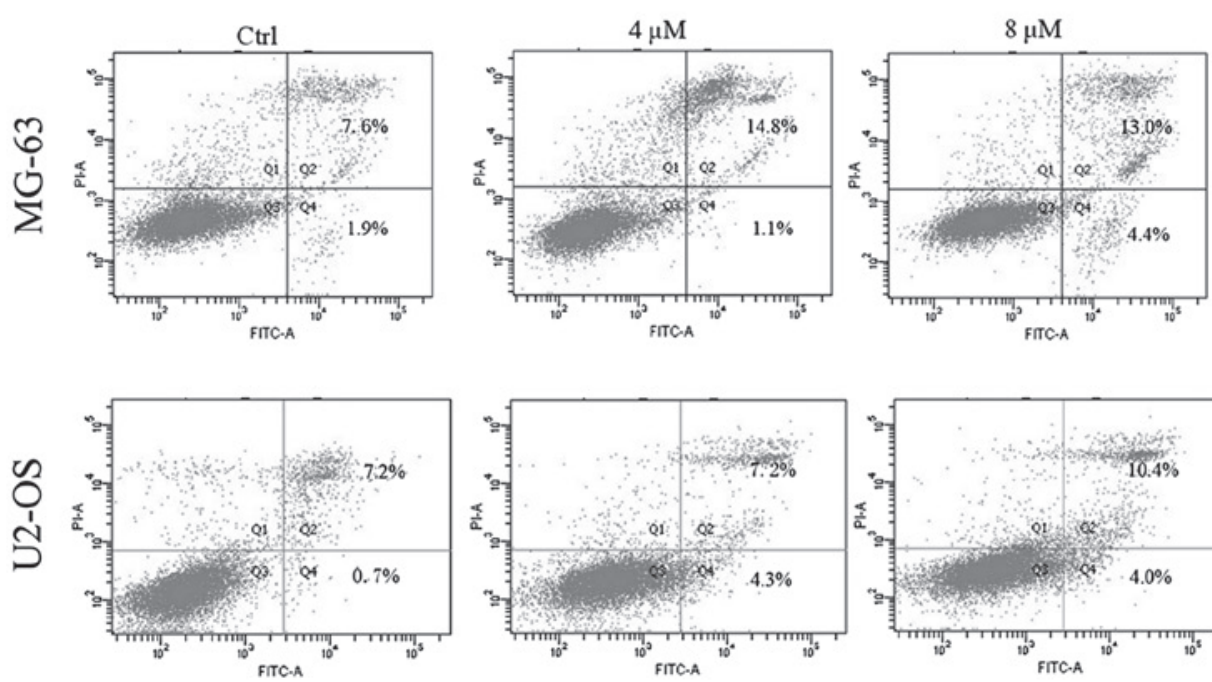

C

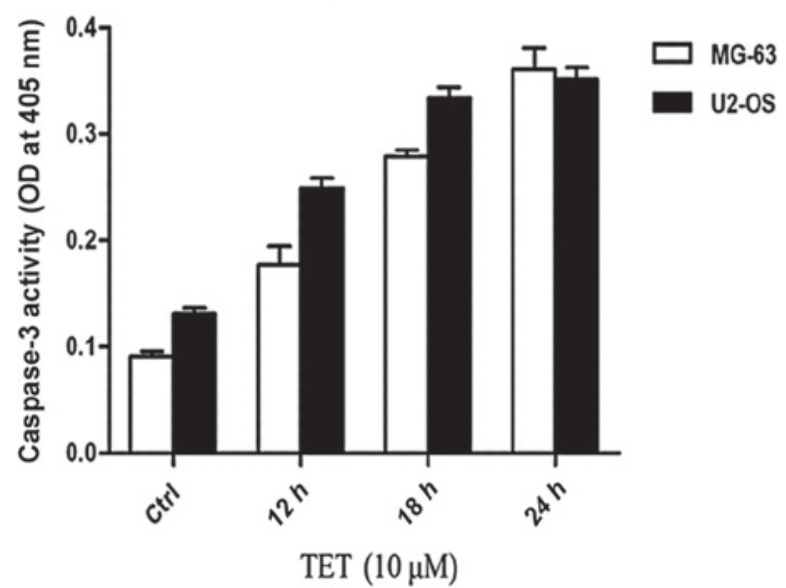

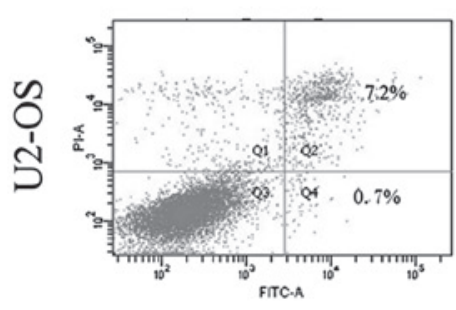

Caspase-3
TET $(10 \mu \mathrm{M})$
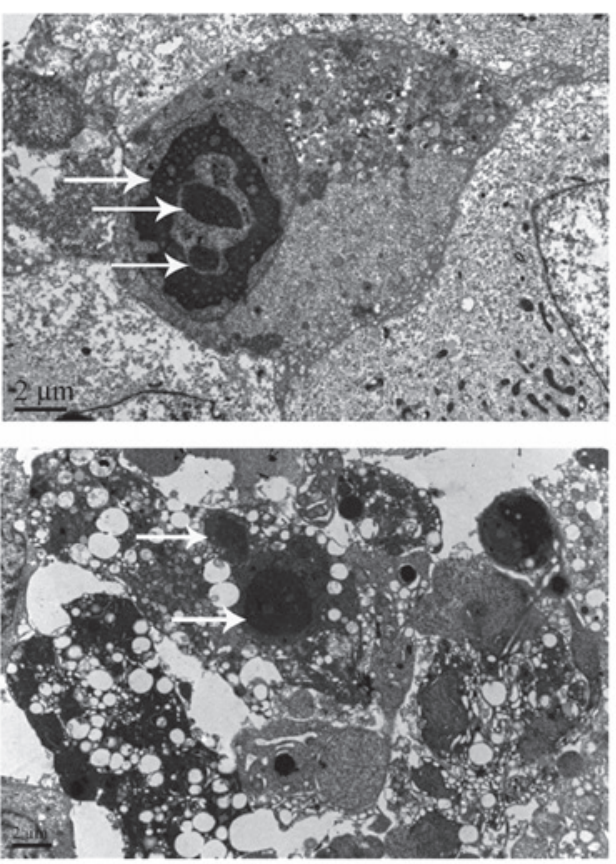
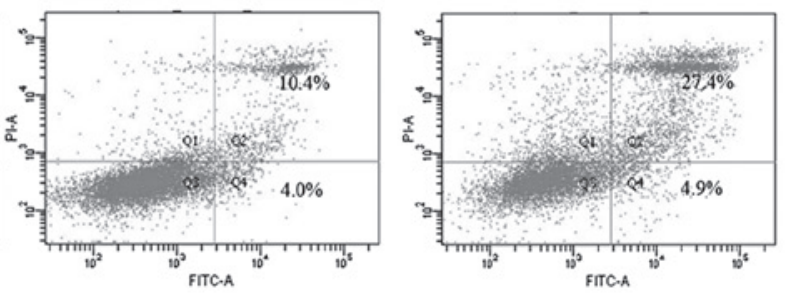

Caspase- 8

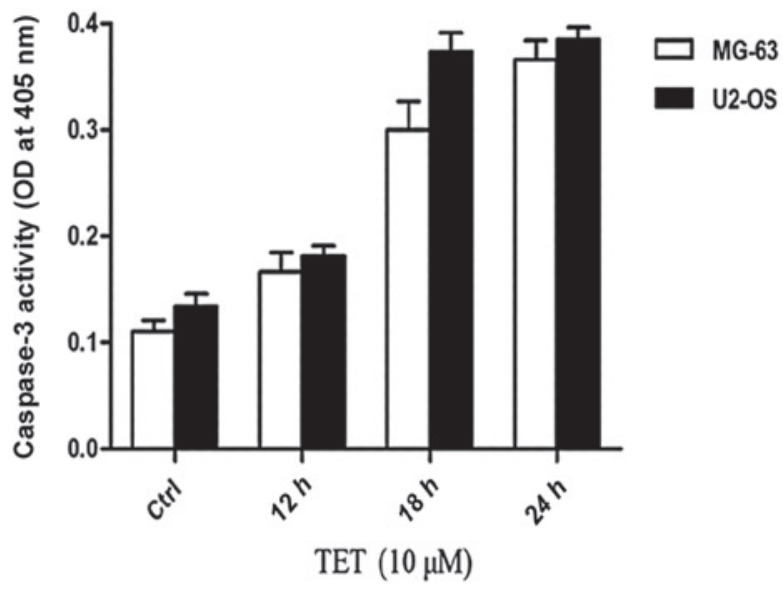

Figure 3. Tetrandrine (TET) induces apoptosis in human osteosarcoma cells. (A) The apoptotic ultrastructure of osteosarcoma cells treated with TET. The cells were examined under a transmission electron microscope (magnification, $\mathrm{x} 3,700$ ). In the $10 \mu \mathrm{M}$ TET group, apoptosis was observed in U-2OS and MG-63 cells. Arrows indicate chromatin condensation and marginalization in MG-63, and fragments of the nucleus and formation of apoptotic bodies in U2-OS cells. Scale bar=2 $\mu \mathrm{m}$. (B) Annexin V-fluorescein isocyanate (FITC)/propidium iodide (PI) staining of osteosarcoma cells treated with varying concentrations of TET. The data are representative of three independent experiments. (C) TET induces the activation of caspase-3 and caspase-8. Lysates were prepared from cells grown in the absence or presence of TET $(10 \mu \mathrm{M})$ for $0,12,18$ or $24 \mathrm{~h}$ and their activities were tested. Data represent the mean \pm SD of three measurements. 
Statistical analysis. All data are expressed as the mean $\pm \mathrm{SD}$. Mean values were calculated from data obtained from experiments performed in triplicate. Student's t-test was used to evaluate the differences between the various experimental and control groups. $\mathrm{IC}_{50}$ values and $95 \%$ confidence intervals (CI) were calculated from MTT assay data using GraphPad Prism 5 (GraphPad Software, Inc., La Jolla, CA, USA). P<0.05 was considered to indicate a statistically significant difference.

\section{Results}

TET inhibits the viability of osteosarcoma cells in a dose-and time-dependent manner. In order to investigate the effects of TET (structure, Fig. 1) on the proliferation of osteosarcoma cells, the growth of MG-63 and U-2OS cells was examined using MTT assays. The results demonstrated that TET inhibited cell proliferation in a dose- and time-dependent manner (Fig. 2). Following incubation with TET for $24 \mathrm{~h}$, the $\mathrm{IC}_{50}$ values for TET were $13.36 \mu \mathrm{M}$ for the MG-63 cells and $9.98 \mu \mathrm{M}$ for the U-2OS cells.

TET induces apoptosis in human osteosarcoma cells. To investigate the mechanism by which TET inhibits the proliferation of human osteosarcoma cells, TEM and flow cytometry analyses were performed. As shown in Fig. 2A, TEM showed typical apoptotic morphological features: Cell shrinkage, nuclear condensation, nuclear fragmentation, chromatin condensation, a crescent-shaped nucleus and cytoplasmic vacuoles. Furthermore, flow cytometry experiments (Fig. 3B) similarly showed that TET is capable of inducing apoptosis in human osteosarcoma cells. These results were consistent with the inhibition of cell growth demonstrated by the MTT assay and suggest that the inhibition of cell viability by TET partly results from the activation of an apoptotic cell-death mechanism. Moreover, a caspase enzyme activity assay revealed that TET caused time-dependent activation of caspase- 3 and caspase- 8 activity (Fig. 3C) at the tested concentration. Thus TET may inhibit the proliferation of human osteosarcoma cells by inducing apoptosis.

TET induces the apoptosis of human osteosarcoma cells through activation of the mitochondrial pathway. Considerable evidence indicates that the mitochondrial pathway is critical in mammalian cell apoptosis. In order to uncover the underlying mechanism by which TET induces the apoptosis of human osteosarcoma cells, a western blot analysis of the signaling pathways involved was performed (Fig. 4). Treatment with TET for $24 \mathrm{~h}$ significantly increased the levels of the pro-apoptotic proteins Bid and Bax, but decreased the levels of the anti-apoptotic proteins $\mathrm{Bcl}-2$ and $\mathrm{Bcl}-\mathrm{xL}$, in a dose-dependent manner. Furthermore, TET increased the protein levels of cytochrome $c$ and Apaf-1. In conclusion, these results suggest that TET induces the apoptosis of human osteosarcoma cells through the activation of the mitochondrial pathway.

\section{Discussion}

In order to improve the 5-year survival rate of osteosarcoma patients, novel strategies and antitumor agents are required. In this study, it was observed that TET dose- and time-dependently inhibited the proliferation of osteosarcoma cells, with $\mathrm{IC}_{50}$

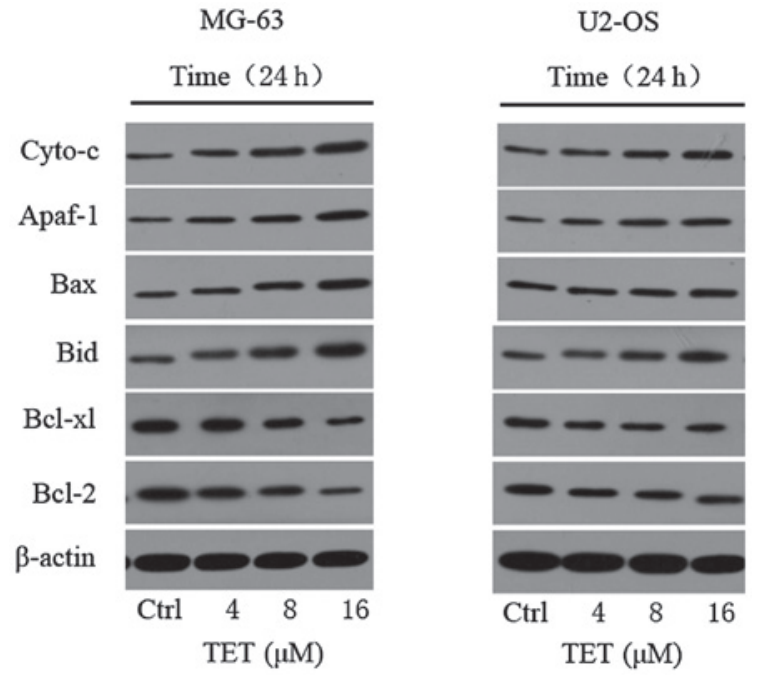

Figure 4. Tetrandrine (TET) upregulates Bid activation and downregulates Bcl-2 and Bcl-xl activation in human osteosarcoma cells in a dose-dependent manner. Western blot analysis of cytochrome $c$ (Cyto-C), apoptic protease-activating factor (Apaf)-1 and Bax in human osteosarcoma cells treated with 4,8 or $16 \mu \mathrm{M}$ TET for $24 \mathrm{~h}$. The densities of target protein bands were analyzed using a Gel-Pro-analyzer (Media Cybernetics, USA). $\beta$-actin served as a loading control.

values in the micromolar range. The $\mathrm{IC}_{50}$ values for TET were $9.98 \mu \mathrm{M}$ for the $\mathrm{U}-2 \mathrm{OS}$ cells and $13.36 \mu \mathrm{M}$ for the MG-63 cells. These values are similar to previously reported $\mathrm{IC}_{50}$ values for other tumor cells, HepG2 $\left(\mathrm{IC}_{50}=9.0 \pm 1.0 \mu \mathrm{M}\right), \mathrm{BGC} 823$ $\left(\mathrm{IC}_{50}=12.28 \pm 1.15 \mu \mathrm{M}\right)$ and $\mathrm{HT} 29$ cells $\left(\mathrm{IC}_{50}=8 \mu \mathrm{M}\right)(14-16)$.

To elucidate the mechanisms underlying the anticancer actions of TET, its pro-apoptotic properties were investigated. Annexin V-FITC/PI double staining revealed that TET induced apoptosis in a dose-dependent manner in the $0-16 \mu \mathrm{M}$ range. These results are consistent with those reported in other cancer cells (15-19). Apoptotic cells were further identified by fluorescence and electron microscopy, which revealed a loss of microvilli, blebbing of the cell surface, condensation of nuclear chromatin and the appearance of apoptotic bodies. These observations are also consistent with those of previous reports $(9,10)$.

Apoptosis occurs via two predominant pathways: The extrinsic and the intrinsic pathways. The extrinsic pathway occurs through the activation of death receptors anchored at the cell membrane by binding to their specific ligands, resulting in the activation of caspase-8. The intrinsic pathway (or mitochondrial pathway) has mitochondria as its central regulator and is associated with the actions of members of the Bcl-2 protein family (20-24). Caspase-3, the most important factor for apoptosis, demonstrated a marked increase following treatment with TET in the present study, and Caspase- 8 , which triggers extrinsic caspase signaling cascades, demonstrated increased activity following treatment with TET for varying lengths of time. These results suggest that TET induces apoptosis in osteosarcoma cells via the extrinsic pathway.

To further clarify the molecular mechanisms, the identification of proteins involved in apoptotic death induced by TET was attempted. Prior to this study, certain studies had indicated that a change in the Bax/Bcl-2 ratio may lead to the release of cytochrome $c$, which binds to Apaf- 1 , resulting in the formation of an apoptosome (25). Bax is a Bcl-2-associated protein with 
pro-apoptotic functions. It is capable of suppressing the ability of Bcl-2 to block apoptosis (26). The activation of Bax following diverse forms of cytotoxic stress has been shown to be an essential gateway to mitochondrial dysfunction and the activation of the intrinsic apoptotic pathway, which is characterized by the release of cytochrome $c$ and the activation of caspase-9/-3 (27). Mitochondrial activation is mediated by $\mathrm{Bid}$, a pro-apoptotic BH3-only Bcl-2 family member. Bid is a substrate of caspase-8, which is activated in low quantities at the death-inducing signaling complex of type II cells (the death-inducing signaling complex of type II cells is very slow and insufficient to directly activate caspase-3). Truncated Bid translocates to the mitochondria and induces loss of mitochondrial membrane potential and the release of apoptogenic factors including cytochrome $c$ (28-30). The overexpression of Bid potentiates apoptosis induced by serum withdrawal (31). Therefore, the protein levels of Bcl-2 family members, including anti-apoptotic molecules including Bcl-2 and Bcl-xl and proapoptotic molecules including Bax and Bid were determined. Furthermore, the protein levels of Cyto-C and Apf-1 were assessed. The results of this study suggest that TET inhibits the growth of human MG-63 and U2-OS cells in vitro by activating the intrinsic pathway (mitochondrial pathway).

In conclusion, although the molecular mechanism underlying the action of TET in tumor cells has not been fully elucidated, these data suggest that TET may directly or indirectly induce apoptosis of osteosarcoma cells not only via the extrinsic pathway, but also the intrinsic pathway. Our data suggest a potential therapy through both the intrinsic and extrinsic pathways with TET for the treatment of osteosarcoma. Further investigations are required to confirm these findings.

\section{Acknowledgements}

This study was supported by grants from the Natural Science Foundation of Zhejiang Province (No. Y206257).

\section{References}

1. Lin YT, Huang AC, Kuo CL, et al: Induction of cell cycle arrest and apoptosis in human osteosarcoma U-2 OS cells by Solanum lyratum extracts. Nutr Cancer 65: 469-479, 2013.

2. Liang CZ, Zhang X, Li H, et al: Gallic acid induces the apoptosis of human osteosarcoma cells in vitro and in vivo via the regulation of mitogen-activated protein kinase pathways. Cancer Biother Radiopharm 27: 701-710, 2012.

3. Cho HJ, Lee TS, Park JB, et al: Disulfiram suppresses invasive ability of osteosarcoma cells via the inhibition of MMP-2 and MMP-9 expression. J Biochem Mol Biol 40: 1069-1076, 2007.

4. Huang G, Mills L and Worth LL: Expression of human glutathione S-transferase P1 mediates the chemosensitivity of osteosarcoma cells. Mol Cancer Ther 6: 1610-1619, 2007.

5. Zhang B, Shi ZL, Liu B, Yan XB, Feng J and Tao HM: Enhanced anticancer effect of gemcitabine by genistein in osteosarcoma: the role of Akt and nuclear factor- $\kappa \mathrm{B}$. Anticancer Drugs 21 288-296, 2010.

6. Posthumadeboer J, van Egmond PW, Helder MN, et al: Targeting JNK-interacting-protein-1 (JIP1) sensitises osteosarcoma to doxorubicin. Oncotarget 3: 1169-1181, 2012.

7. Zhang Y, Wei RX, Zhu XB, Cai L, Jin $\mathrm{W}$ and $\mathrm{Hu} \mathrm{H}$ : Tanshinone IIA induces apoptosis and inhibits the proliferation, migration, and invasion of the osteosarcoma MG-63 cell line in vitro. Anticancer Drugs 23: 212-219, 2012.

8. Wu HY, Lin TK, Kuo HM, et al: Phyllanthus urinaria induces apoptosis in human osteosarcoma 143B cells via activation of Fas/FasL- and mitochondria-mediated pathways. Evid Based Complement Alternat Med 2012: 1-13, 2012.
9. Teh BS, Chen P, Lavin MF, Seow WK and Thong YH: Demonstration of the induction of apoptosis (programmed cell death) by tetrandrine, a novel anti-inflammatory agent. Int J Immunopharmacol 13: 1117-1126, 1991.

10. Lai YL, Chen YJ, Wu TY, et al: Induction of apoptosis in human leukemic U937 cells by tetrandrine. Anticancer Drugs 9: 77-81, 1998.

11. Dong Y, Yang MM and Kwan CY: In vitro inhibition of proliferation of HL-60 cells by tetrandrine and coriolus versicolor peptide derived from Chinese medicinal herbs. Life Sci 60: PL135-140, 1997.

12. Cho HS, Chang SH, Chung YS, et al: Synergistic effect of ERK inhibition on tetrandrine-induced apoptosis in A549 human lung carcinoma cells. J Vet Sci 10: 23-28, 2009.

13. He BC, Gao JL, Zhang BQ, et al: Tetrandrine inhibits Wnt/ $\beta$-catenin signaling and suppresses tumor growth of human colorectal cancer. Mol Pharmacol 79: 211-219, 2011.

14. Yoo SM, Oh SH, Lee SJ, et al: Inhibition of proliferation and induction of apoptosis by tetrandrine in HepG2 cells. J Ethnopharmacol 81: 225-229, 2002.

15. Wei J, Liu B, Wang L, Qian X, Ding Y and Yu L: Synergistic interaction between tetrandrine and chemotherapeutic agents and influence of tetrandrine on chemotherapeutic agent-associated genes in human gastric cancer cell lines. Cancer Chemother Pharmacol 60: 703-711, 2007.

16. Meng LH, Zhang H, Hayward L, Takemura H, Shao RG and Pommier Y: Tetrandrine induces early G1 arrest in human colon carcinoma cells by down-regulating the activity and inducing the degradation of G1-S-specific cyclin-dependent kinases and by inducing p53 and p21Cip1. Cancer Res 64: 9086-9092, 2004.

17. Zhang Y, Wang C, Wang $\mathrm{H}$, Wang $\mathrm{K}$, Du $\mathrm{Y}$ and Zhang J: Combination of tetrandrine with cisplatin enhances cytotoxicity through growth suppression and apoptosis in ovarian cancer in vitro and in vivo. Cancer Lett 304: 21-32, 2011.

18. Liu C, Gong K, Mao X and Li W: Tetrandrine induces apoptosis by activating reactive oxygen species and repressing Akt activity in human hepatocellular carcinoma. Int J Cancer 129: 1519-1531, 2011.

19. Li X, Su B, Liu R, Wu D and He D: Tetrandrine induces apoptosis and triggers caspase cascade in human bladder cancer cells. J Surg Res 166: e45-51, 2011.

20. Lima RT, Busacca S, Almeida GM, Gaudino G, Fennell DA and Vasconcelos MH: MicroRNA regulation of core apoptosis pathways in cancer. Eur J Cancer 47: 163-174, 2011.

21. Fernandez-Luna JL: Regulation of pro-apoptotic BH3-only proteins and its contribution to cancer progression and chemoresistance. Cell Signal 20: 1921-1926, 2008.

22. Liang CZ, Zhang JK, Shi Z, Liu B, Shen CQ and Tao HM: Matrine induces caspase-dependent apoptosis in human osteosarcoma cells in vitro and in vivo through the upregulation of Bax and Fas/FasL and downregulation of Bcl-2. Cancer Chemother Pharmacol 69: 317-331, 2011.

23. Ito Y, Pandey P, Sporn MB, Datta R, Kharbanda S and Kufe D: The novel triterpenoid CDDO induces apoptosis and differentiation of human osteosarcoma cells by a caspase- 8 dependent mechanism. Mol Pharmacol 59: 1094-1099, 2001.

24. Oh SH and Lee BH: Induction of apoptosis in human hepatoblastoma cells by tetrandrine via caspase-dependent Bid cleavage and cytochrome c release. Biochem Pharmacol 66: 725-731, 2003.

25. Shukla RK, Kumar A, Gurbani D, Pandey AK, Singh S and Dhawan $\mathrm{A}$ : $\mathrm{TiO}_{2}$ nanoparticles induce oxidative DNA damage and apoptosis in human liver cells. Nanotoxicology 7: 48-60, 2013.

26. Kaseta MK, Khaldi L, Gomatos IP, et al: Prognostic value of bax, bcl-2, and p53 staining in primary osteosarcoma. J Surg Oncol 97: 259-266, 2008

27. Cao X, Bennett RL and May WS: c-Myc and caspase-2 are involved in activating Bax during cytotoxic drug-induced apoptosis. J Biol Chem 283: 14490-14496, 2008.

28. Schmitz I, Kirchhoff S and Krammer PH: Regulation of death receptor-mediated apoptosis pathways. Int J Biochem Cell Biol 32: 1123-1136, 2000.

29. Luo X, Budihardjo I, Zou H, Slaughter C and Wang X: Bid, a $\mathrm{Bcl} 2$ interacting protein, mediates cytochrome $c$ release from mitochondria in response to activation of cell surface death receptors. Cell 94: 481-490, 1998.

30. Liu X, Kim CN, Yang J, Jemmerson R and Wang X: Induction of apoptotic program in cell-free extracts: requirement for dATP and cytochrome $c$. Cell 86: 147-157, 1996.

31. Wang K, Yin XM, Chao DT, Milliman CL and Korsmeyer SJ: BID: a novel BH3 domain-only death agonist. Genes Dev 10: 2859-2869, 1996 . 